mainly in the state 2. The deflection experiments with para-hydrogen at room temperature allow, therefore, the determination of the rotational moment, which has been found to be between 0.8 and 0.9 nuclear magnetons per unit quantum number.

In the case of ortho-hydrogen, the lowest rotational quantum state possible is the state 1 . Therefore, even at the lowest temperatures, the rotational magnetic moment is superimposed on that due to the two protons with parallel spin. Since, however, the rotational moment is known from the experiments with pure para-hydrogen, the moment of the protons can be determined from deflection experiments with ortho-hydrogen, or with ordinary hydro. gen consisting of 75 per cent ortho-and 25 per cent para-hydrogen. The value obtained is 5 nuclear magnetons for the two protons in the ortho-hydrogen molecule, that is, $\mathbf{2} \cdot 5$ (and not 1) nuclear magnetons for the proton.

This is a very striking result, but further experiments carried out with increased accuracy and over a wide range of experimental conditions (such as temperature, width of beam, etc.) have. shown that it is correct within a limit of less than 10 per cent.

A more detailed account of these experiments will appear in the Zeitschrift für Physik.

I. EstermanN.

R. Frisch.

O. STERN.

Institut für physikalische Chemie,

Hamburgischer Universitët. June 19.

\section{Gravitational Field of an Electron}

IN 1926, Einstein suggested ${ }^{1}$ that the gravitational equations in an electromagnetie field should be taken as

$$
K_{p q}-\frac{1}{4} g_{p q} K=-8 \pi E_{p q},
$$

where $E_{p q}$ is the electromagnetic energy-tensor.

The solution of this equation for the radially symmetric field

$$
d s^{2}=-e^{\lambda} d r^{2}-r^{2} d \theta^{2}-r^{2} \sin ^{2} \theta d \varphi^{2}+e^{\nu} d t^{2}
$$

is found to be

$$
e^{-\lambda}=e^{\nu}=1-\frac{1}{3} \alpha r^{2}-\frac{2 m}{r}+\frac{4 \pi \varepsilon^{2}}{r^{2}},
$$

where $\alpha, m, \varepsilon$ are constants of integration. The constants $m$ and $\varepsilon$ may be interpreted (as in the well-known solutions of Nordström and Jeffery) as the mass and the charge of the electron respectively. But it is difficult to interpret the second term. The most probable interpretation is that the empty space-time is de Sitterean and not Galilean. The details of the solution will appear shortly.

Presidency College,

J. GHosh. Calcutta.

${ }^{1}$ Mathematische Annalen, Dec., 1826.

\section{A Thermal Reaction between Chlorine and Formaldehyde}

It has been known for many years that chlorine reacts with the solid polymers of formaldehyde in the dark to give carbon monoxide and hydrogen chloride ${ }^{1}$. Furthermore, rapid reaction occurs in strong sunlight resulting in the formation of phosgen $\theta^{t, 2}$.

We have carried out some experiments with pure gaseous formaldehyde ${ }^{3}$ and find that no measureable reaction takes place at $100^{\circ}$. At $150^{\circ}$, however, after a short induotion period; a rapid reaction sets in accompanied by an increase in pressure. In such an experiment, starting with $191 \mathrm{~mm}$, of formaldehyde and $183 \mathrm{~mm}$. of chlorine, the pressure increased $148 \mathrm{~mm}$. in 48 minutes. The reaction vessel had a volume of 156.5 c.c. and the dead space was reduced to very small dimensions by the use of a quartz spiral manometer. A known fraction of the gas was removed at this stage and cooled in a liquid air trap to condense the chlorine, formaldehyde and hydrogen chloride. The carbon monoxide which remained was collected by an automatic Töpler pump and analysed in a Bone and Wheeler apparatus. The condensate was dissolved in potassium iodide solution and the hydrochloric acid determined by titration, after removing the liberated iodine with thiosulphate. According to these analyses, 23.4 c.c. carbon monoxide and 44.8 c.c. hydrogen chloride at N.T.P. were formed in the reaction vessel. Both values are slightly greater than the theoretical amounts corresponding to a pressure increase of $148 \mathrm{~mm}$., due to the fact that the induction period was extremely short in this case and some reaction had occurred before the first pressure reading could be taken.

However, it is apparent from the above as well as from other data not enumerated here that the reaction proceeds in the dark according to the following equation:

$$
\mathrm{HCHO}+\mathrm{Cl}_{2} \rightarrow \mathrm{CO}+2 \mathrm{HCl}
$$

It is probable that a complex chain process is involved and a detailed study of the kinetics is in progress, with a view to further elucidation of the mechanism.

R. SPEnCE

W. WILD.

Physical Chemistry Department,

The University, Leeds, June 27.

1 A. Brochet, C. R., 121, 1156 ; 1895.

w. Tishtchenko, J. Russ. Chem. Soc., 479; 1887

s M. Trautz and E. U fer, $J$. pr. Chem., 113, $105 ; 1926$.

\section{Origin of Monochromatic Radiation}

IT is a remarkable fact that a particle possessing two degrees of freedom of rotation in co-latitude $\theta$ and azimuth $\varphi$, where $\dot{\theta}$ and $\phi$ vary in a continuous manner, may give a stationary projection on the equatorial plane relatively to axes rotating in this plane with a certain uniform angular velocity.

Let $\dot{\theta}=\dot{\theta}_{1}+\left(\dot{\theta}_{0}-\dot{\theta}_{1}\right) e^{-\alpha t} ; \dot{\varphi}=\dot{\varphi}_{1} \rightarrow\left(\dot{\varphi}_{1}-\dot{\varphi}_{0}\right) e^{-\alpha t}$ where $\hat{\theta}_{0} \dot{\varphi}_{0}, \dot{\theta}_{1} \dot{\varphi}_{1}$ are the initial and final angular velocities respectively. Using the relations $\theta=\int \dot{\theta} d t, \varphi=\int \dot{\phi} d t$, it then appears that the projected path on the equatorial plane $O X Y$ will be

$$
r=a \sin \left\{\left(\frac{\dot{\theta}_{0} \dot{\varphi}_{1}-\dot{\theta}_{1} \dot{\varphi}_{0}}{\dot{\theta}_{0}-\dot{\theta}_{1}}\right) t-\varphi\right\} \frac{\dot{\theta}_{0}-\dot{\theta}_{1}}{\dot{\varphi}_{1}-\dot{\varphi}_{0}}
$$

$\alpha$ has disappeared and relatively to axes rotating about $O Z$ with a uniform angular velocity given by the coefficient of $t$ within the bracket, the projected path will be

$$
r=-a \sin \left(\frac{\dot{\theta}_{0}-\hat{\theta}_{2}}{\dot{\varphi}_{1}-\dot{\phi}_{0}}\right) \phi ;
$$

curiously enough, this relative path will appear stationary if the total fall of one angular velocity in latitude bears any simple relation to the rise of the other in azimuth.

Perhaps it is too ambitious to try to find some 'Victorian' mechanism to explain what has remained unassailed as a postulate for the past twenty years, 